\title{
Detection of Cancer in Medical Images using Deep Learning
}

\author{
https://doi.org/10.3991/ijoe.v17i14.27349
}

Emad Shweikeh ${ }^{1(\bowtie)}$, Joan Lu' ${ }^{1}$, Murad Al-Rajab ${ }^{2}$

${ }^{1}$ University of Huddersfield, Huddersfield, England

${ }^{2}$ Abu Dhabi University, Abu Dhabi, United Arab Emirates

Emad.Shweikeh@hud.ac.uk

\begin{abstract}
Cancer is a serious disease that causes death by genomic disorder combination and diversity of unreasoning changes. This paper study the major deep learning techniques that are addressing medical image analysis and summarizes over 200 contributed articles to the subject, in particular those studies that are published in the last 6 years (since 2016). The main purpose of this paper study is to survey the deep learning algorithms for cancer detection and diagnosis. the results show that the convolutional neural network $(\mathrm{CNN})$ is the most broadly used when it comes to deep learning and medical image analysis.
\end{abstract}

Keywords - machine learning (ML), deep learning (DL), image analysis, convolution neural network $(\mathrm{CNN})$, cancer detection, survey

\section{Introduction}

A tumor formed from the abnormality of organs regularly with fast development which is the main cause of lost life worldwide [1]. As per GLOBOCAN numbers and statistics, approximately $18.1 \mathrm{M}$ new cancers cases occurred in 2018 which leads to $9.6 \mathrm{M}$ cancer losses [2,3]. As per the report, the leader cancer is lung cancer a cause of death (18.4 percent), next is breast (6.6 percent), colon (5.8 percent), prostate (3.8 percent), and skin (1.3 percent) including melanoma and non-melanoma [1, 4]. It is also noted that Asia accounts for more than half of all cancer deaths, while Europe accounts for 20.3 percent of all cancer deaths [5]. Medical Imaging Analysis plays a vital part in detecting anomalies in various organs of the body, for example, breast cancer, lung cancer, brain tumor, skin cancer, prostate cancer, and colon cancer [2, 3, and 4].

Furthermore, medical images are an important method to diagnose disease, that helps the physicians to instinctively observe a patient's body organs and professionally examine the illness possibility [1]. Medical Service providers usually produce for each patient hundreds of medical images, so it is a great opportunity as well as a challenge to digest, process then analyzes the huge amount of medical image data [5]. 


\section{Research approaches}

Deep learning is the main contributor to the recent and present-day studies of Artificial Intelligence in almost all sides of life [6]. This study objective is to provide a survey on the machine learning techniques and more specifically on the most common deep learning algorithms which are implemented for the diagnosis and detection of tumor [7, 8, 9, and 10]. In this study, we summarize more than 200 articles and contributions to the field in the last 5 years since 2016. Furthermore, in this research study, the study includes a brief of deep learning and the popular frameworks addressing the diagnose of cancer.

The research methods and techniques are listed in the following steps as explained in Figure 1.

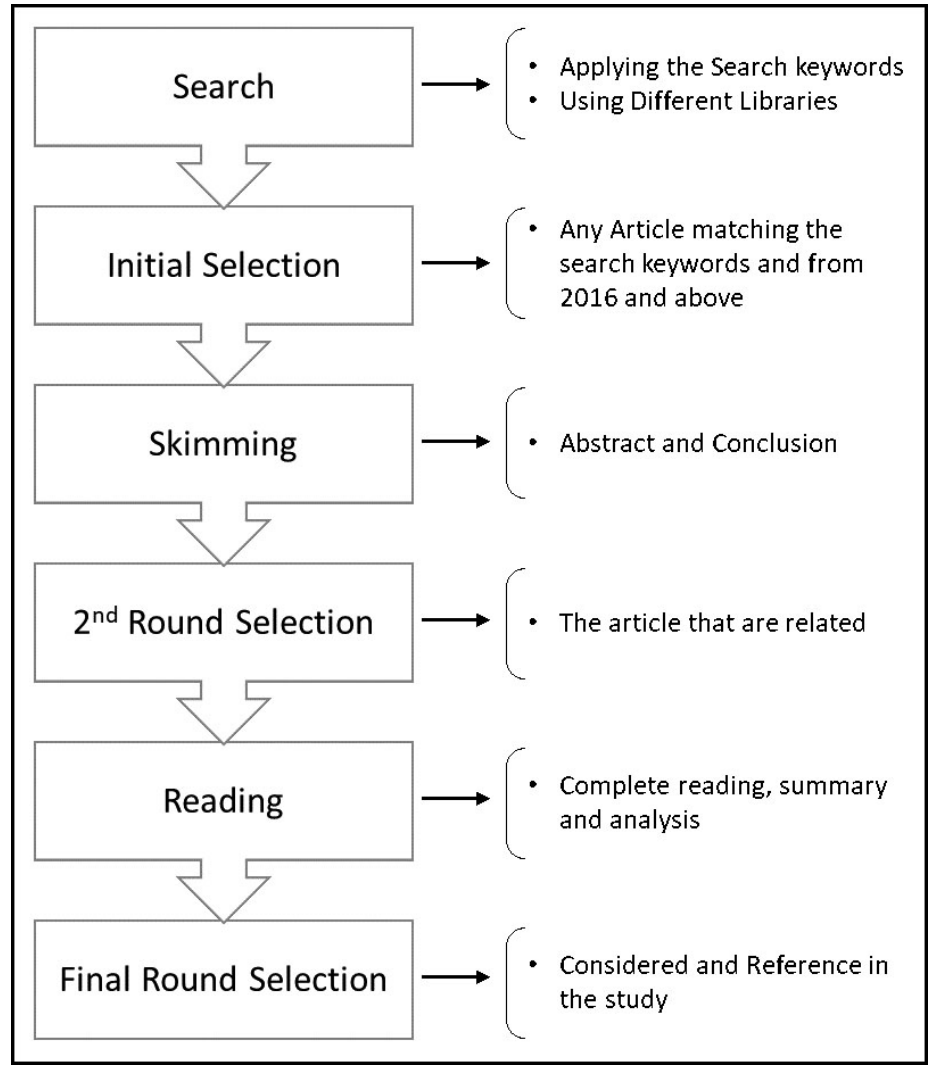

Fig. 1. Search methodology

- The selection of studies and papers was at first created by looking for the search results in different search engines, global information analytics library, technical professional organization library, such as (Google Scholar, IEEE, Elsevier, ...etc.). the searches for terms "Deep Learning", "Classification", "Image Enhancement", 
"Segmentation", and "Survey" for medical imaging papers as listed in Table 1. This study is including only papers that are available online from 2016 onward.

- The study focused on a summary of the related content and results that is as sociated with the purpose of this study.

- A systematic review of the latest studies (last 6 years) of different types of cancers has been conducted. The research lists the Deep Learning Algorithms that are related to Medical Images Analysis and Cancer Detection.

- Studying and analyzing the selected papers that are fitting within the scope of the work and matching with the keywords as per Table 1 and published from 2016, as a result, 91 articles have been considered, the researcher highlights main four cancers that this study is focusing on. Figure 1 is explaining the search methodology for the study.

- Classifying the Studied papers by algorithms associated or used for detection of each cancer. Important note that in case of similarity of many papers, a quick comparison between these papers is conducted to identify the useful one for this study in case not easy to do so, only the recent paper was included. The paper is organized to starts with a general overview of Machine Learning and Related Medical Images studies. Then the paper introduces the general overview of deep learning. This is followed by a highlight of the latest studies in the field, highlighting progress related to each cancer and associated Algorithm for cancer detection, at the end, we include a brief description and remarks on the recent work on tumor diagnosis and classification of deep learning and propose some future research directions. the paper concluded with a brief regarding the future of deep learning in particular cancer. This study is organized based on types of cancer.

Table 1. The keywords that are used in the search

\begin{tabular}{|l|l|l|}
\hline \multicolumn{2}{|c|}{ Keywords } \\
\hline Medical Images & Deep Learning - DL & Image Enhancement \\
\hline Classification & Segmentation & Image Detection \\
\hline Survey & Cancer & Image Analysis \\
\hline
\end{tabular}

\section{$3 \quad$ Results and discussion}

A structured review of works of literature classifies the most important scientific contributions in a particular field. This study covers the latest research on applying ML/DL for cancer detection and diagnosis. Table 2 summarizes algorithms associated with which cancer type it was employed. It is found that CNN and Deep Convolutional Neural Network (DCNN) algorithms have been selected as the implemented algorithm for most cancer types, while Support-Vector Machines (SVM) algorithm comes second in a row. 
Table 2. Cancer types associated with algorithms

\begin{tabular}{|l|c|c|c|c|c|c|c|c|c|c|c|}
\hline Algorithm & ANN & CNN & $\begin{array}{c}\text { CNN } \\
\text { \& } \\
\text { SVM }\end{array}$ & DBN & DCNN & FCRN & KNN & $\begin{array}{c}\text { RF } \\
\text { CNN } \\
\& \\
\text { ANN }\end{array}$ & SSAE & SVM & $\begin{array}{c}\text { SVM } \\
\text { \& } \\
\text { CNN }\end{array}$ \\
\hline Breast & & $\checkmark$ & & $\checkmark$ & $\checkmark$ & & $\checkmark$ & $\checkmark$ & & $\checkmark$ & \\
\hline Colon & & $\checkmark$ & $\checkmark$ & & & & & & & & $\checkmark$ \\
\hline Prostate & & $\checkmark$ & & & $\checkmark$ & & & & $\checkmark$ & & \\
\hline Skin & $\checkmark$ & $\checkmark$ & & & $\checkmark$ & $\checkmark$ & & & & $\checkmark$ & \\
\hline
\end{tabular}

Table 3. Evidence of studies associated with algorithms used for cancer research

\begin{tabular}{|l|l|c|}
\hline \multicolumn{1}{|c|}{$\begin{array}{c}\text { Machine Learning } \\
\text { Technique }\end{array}$} & \multicolumn{1}{|c|}{ Reference } & $\begin{array}{c}\text { Total Number of } \\
\text { Studies }\end{array}$ \\
\hline CNN & $1,3,5,7,10,11,13,14,17,18,19,20,21,23$, & 35 \\
& $24,25,26,27,28,29,30,31,32,33,34,37,38$, \\
& $40,41,42,43,44,47,48,49,50,52$ & \\
\hline ANN & 36 & 2 \\
\hline DBN & 13 & 1 \\
\hline KNN & 12,16 & 2 \\
\hline Hybrid & $15,45,46$ & 3 \\
\hline Others & $2,11,22,35,38$ & 5 \\
\hline
\end{tabular}

Table 2 and Table 3 shows that CNN is the most popular algorithm applied for cancer research. Other algorithms are very likely to become future candidates, but they are not often reported.

It is obvious that deep learning will be very essential in many applications of medical images and will play an important and essential factor in the future practice of medical images (Radiology, MRI, CT SCAN, etc. ...) as addressed in [12]. The prediction using deep learning algorithms will execute routine tasks, while physicians focus on the core work as addressed by [1]. The deep learning algorithms will work tightly and closely to bring a high and accurate performance that is indicated by $[13,14,15$ and 16]. Some prediction using deep learning algorithms will substitute physicians altogether - at least in their image explanation side- [17,18,19 and 20]. It follows that deep learning has exposed astonishing potential in many tasks that are image-related, but not enough results in the field [21]. A small number of recent studies highlight that the act of the algorithms equivalent to human's expert in similar tasks [7 and 22].

\section{Conclusion and future work}

The goal of this research is to combine Convolutional Neural Networks (CNNs), Long-Short Term Memory (LSTMs), and Auto-encoders (AEs) in order to improve credit card fraud detection and the performance of prior models. CNN, AE, LSTM, and 
AE\&LSTM are four models that can be used. Different parameter values are used to train each of these models. The AE model has the highest accuracy, with an accuracy of 0.99 , the CNN model has an accuracy of 0.85 , the LSTM model has an accuracy of 0.85 , and the AE\&LSTM model has an accuracy of 0.32 after 400 epochs. The AE classifies the best outcome among these models, it is concluded.

\section{$5 \quad$ References}

[1] Bray, F., Ferlay, J., Soerjomataram, I., Siegel, R. L., Torre, L. A., \& Jemal, A. (2018). Global cancer statistics 2018: GLOBOCAN estimates of incidence and mortality worldwide for 36 cancers in 185 countries. CA: a cancer journal for clinicians, 68: 394-424. https://doi. org/10.3322/caac. 21492

[2] Ghavami, N., Hu, Y., Gibson, E., Bonmati, E., Emberton, M., Moore, C. M., \& Barratt, D. C. (2019). Automatic segmentation of prostate MRI using convolutional neural networks: Investigating the impact of network architecture on the accuracy of volume measurement and MRI-ultrasound registration. Medical image analysis, 58: 101-558. https://doi. org/10.1016/j.media.2019.101558

[3] Maa, L., Guoa, R., Zhanga, G. M., Tadea, F., Schustera, D. M., Niehc, P., Masterc, V., \& Fei, B., (2017). Automatic segmentation of the prostate on CT images using deep learning and multi-atlas fusion, SPIE Medical Imaging, International Society for Optics and Photonics. https://doi.org/10.1117/12.2255755

[4] Chowdhury, A., Sevinsky, C. J., Santamaria-Pang, A., \& Yener, B. A computational study on convolutional feature combination strategies for grade classification in colon cancer using fluorescence microscopy data, in: Proceedings of SPIE, 2017, p. 10140. https://doi. org/10.1117/12.2255687

[5] Al-Antari, M. A., Han, S. M., \& Kim, T. S. (2020). Evaluation of deep learning detection and classification towards computer-aided diagnosis of breast lesions in digital X-ray mammograms. Computer methods and programs in biomedicine, 196: 105-584. https://doi. org/10.1016/j.cmpb.2020.105584

[6] Hussein, S., Kandel, P., Bolan, C. W., Wallace, M. B., \& Bagci, U. (2019). Lung and pancreatic tumor characterization in the deep learning era: novel supervised and unsupervised learning approaches. IEEE transactions on medical imaging, 38: 1777-1787. https://doi. org/10.1109/TMI.2019.2894349

[7] Shaikh, A. (2015). The impact of SOA on a system design for a telemedicine healthcare system. Network Modeling Analysis in Health Informatics and Bioinformatics, 4(1), 1-16. https://doi.org/10.1007/s13721-015-0087-0

[8] Al-Rajab, M., \& Lu, J. (2016). A study on the most common algorithms implemented for cancer gene search and classifications. International Journal of Data Mining Bioinformatics, 14: 159-176. https://doi.org/10.1504/IJDMB.2016.074685

[9] Al-Rajab, M., Lu, J., \& Xu, Q. (2021). A framework model using multifilter feature selection to enhance colon cancer classification. Plos one, 16:e249094. https://doi.org/10.1371/ journal.pone.0249094

[10] Lu, Z., Xu, Q., Al-Rajab, M., \& Chiazor, L. (2021). Machine Learning in Cancer Research With Applications in Colon Cancer and Big Data Analysis. IGI Global. Khatri, S., Arora, A., \& Arun Agrawal, P., Supervised machine learning algorithms for credit card fraud detection: a comparison. $10^{\text {th }}$ International Conference on Cloud Computing, Data Science \& Engineering (Confluence), January 29 2020, IEEE, pp. 680-683. https://doi. org/10.4018/978-1-7998-7316-7 
[11] Quasim, M. T., Alhuwaimel, S., Shaikh, A., Asiri, Y., Rajab, K. et al. (2021). An Improved Machine Learning Technique with Effective Heart Disease Prediction System. CMCComputers, Materials \& Continua, 69: 4169-4181. https://doi.org/10.32604/ cmc.2021.015984

[12] Kwak, J. T., \& Hewitt, S. M. (2017). Lumen-based detection of prostate cancer via convolutional neural networks. In Medical Imaging 2017: Digital Pathology 10140: 1014008. https://doi.org/10.1117/12.2253513

[13] Mazurowski, M. A., Buda, M., Saha, A. \& Bashir, M. R. (2019), Deep learning in radiology: An overview of the concepts and a survey of the state of the art with focus on MRI. J. Magn. Reson. Imaging, 49: 939-954. https://doi.org/10.1002/jmri.26534

[14] Chen, M., Shi, X., Zhang, Y., Wu, D., \& Guizani, M. (2017). Deep features learning for medical image analysis with convolutional autoencoder neural network. IEEE Transactions on Big Data.

[15] Bardou, D., Zhang, K., \& Ahmad, S. M. (2018). Classification of breast cancer based on histology images using convolutional neural networks. Ieee Access, 6: 24680-24693. https:// doi.org/10.1109/ACCESS.2018.2831280

[16] Dhahri, H., Al Maghayreh, E., Mahmood, A., Elkilani, W., \& Faisal Nagi, M. (2019). Automated breast cancer diagnosis based on machine learning algorithms. Journal of healthcare engineering, 2019: 1-11. https://doi.org/10.1155/2019/4253641

[17] Htay, T. T., \& Maung, S. S. Early stage breast cancer detection system using glcm feature extraction and k-nearest neighbor (k-NN) on mammography image. In 2018 18th International Symposium on Communications and Information Technologies (ISCIT), September 2018, IEEE, pp. 171-175. https://doi.org/10.1109/ISCIT.2018.8587920

[18] Saeed, S., Shaikh, A., Memon, M. A., Saleem, M. Q., \& Naqvi, S. M. R. (2017). Assessment of Brain Tumor Due to the Usage of MATLAB Performance. Journal of Medical Imaging and Health Informatics, 7(6), 1454-1460. https://doi.org/10.1166/jmihi.2017.2187

[19] Bareiro Paniagua, L. R., Leguizamón Correa, D. N., Pinto-Roa, D. P., Vázquez Noguera, J. L., \& Salgueiro Toledo, L. A. (2016). Computerized Medical Diagnosis of Melanocytic Lesions based on the ABCD approach. CLEI Electronic Journal, 19: 6-6. https://doi. org/10.19153/cleiej.19.2.5

[20] Lo, S. B., Chan, H., Lin, J., Li, Matthew T. F., and Mun, K. S. (1995). Artificial convolution neural network for medical image pattern recognition, Neural Networks, 8: 1201-1214. https://doi.org/10.1016/0893-6080(95)00061-5

[21] Gong, C., Tao, D., Maybank, S. J., Liu, W., Kang, G., \& Yang, J. (2016). Multi-modal curriculum learning for semi-supervised image classification. IEEE Transactions on Image Processing, 25: 3249-3260. https://doi.org/10.1109/TIP.2016.2563981

[22] Miotto, R., Wang, F., Wang, S., Jiang, X., \& Dudley, J. T. (2018). Deep learning for healthcare: review, opportunities and challenges. Briefings in bioinformatics, 19: 1236-1246. https://doi.org/10.1093/bib/bbx044

[23] Abdel-Zaher, A. M., \& Eldeib, A. M. (2016). Breast cancer classification using deep belief networks. Expert Systems with Applications, 46: 139-144. https://doi.org/10.1016/j. eswa.2015.10.015

[24] Hou, D., Hou, R., \& Hou, J. On-device Training for Breast Ultrasound Image Classification. In 2020 10th Annual Computing and Communication Workshop and Conference (CCWC), January 2020, IEEE, pp. 0078-0082. https://doi.org/10.1109/CCWC47524.2020.9031146

[25] Saeed, S., Shaikh, A., Memon, M. A., \& Naqvi, S. M. R. (2018). Impact of data mining techniques to analyze health care data. Journal of Medical Imaging and Health Informatics, 8(4), 682-690. https://doi.org/10.1166/jmihi.2018.2385

[26] Halim, E., Halim, P. P., \& Hebrard, M. Artificial intelligent models for breast cancer early detection. In 2018 International Conference on Information Management and Technology (ICIMTech), September 2018, IEEE, pp. 517-521. https://doi.org/10.1109/ ICIMTech.2018.8528140 
[27] Swiderski, B., Kurek, J., Osowski, S., Kruk, M., \& Barhoumi, W. Deep learning and non-negative matrix factorization in recognition of mammograms. In Eighth International Conference on Graphic and Image Processing (ICGIP 2016) February 2017 (Vol. 10225, p. 102250B). https://doi.org/10.1117/12.2266335

[28] Spanhol, F. A., Oliveira, L. S., Petitjean, C., \& Heutte, L. Breast cancer histopathological image classification using convolutional neural networks. In 2016 international joint conference on neural networks (IJCNN), July 2016, IEEE, pp. 2560-2567. https://doi.org/10.1109/ IJCNN.2016.7727519

[29] Khan, S., Islam, N., Jan, Z., Din, I. U., \& Rodrigues, J. J. C. (2019). A novel deep learning based framework for the detection and classification of breast cancer using transfer learning. Pattern Recognition Letters, 125: 1-6. https://doi.org/10.1016/j.patrec.2019.03.022

[30] Chougrad, H., Zouaki, H., \& Alheyane, O. (2018). Deep convolutional neural networks for breast cancer screening. Computer methods and programs in biomedicine, 157: 19-30. https://doi.org/10.1016/j.cmpb.2018.01.011

[31] Yadav, S. S., \& Jadhav, S. M. Deep convolutional neural network based medical image classification for disease diagnosis. J Big Data 6, 113 (2019). https://doi.org/10.1186/ s40537-019-0276-2

[32] Guo, Y., Gao, Y., \& Shen, D. (2015). Deformable MR prostate segmentation via deep feature learning and sparse patch matching. IEEE transactions on medical imaging, 35: 1077-1089. https://doi.org/10.1109/TMI.2015.2508280

[33] Gupta, J., Saini, S. K., \& Juneja, M. (2020). Survey of denoising and segmentation techniques for MRI images of prostate for improving diagnostic tools in medical applications. Materials Today: Proceedings, 28: 1667-1672. https://doi.org/10.1016/j.matpr.2020.05.023

[34] K. Yan et al. "Automatic prostate segmentation on MR images with deep network and graph model," 2016 38th Annual International Conference of the IEEE Engineering in Medicine and Biology Society (EMBC), Orlando, FL, 2016, pp. 635-638. https://doi.org/10.1109/ EMBC.2016.7590782

[35] Tian, Z., Liu, L., \& Fei, B. (2017, March). Deep convolutional neural network for prostate MR segmentation. In Medical Imaging 2017: Image-Guided Procedures, Robotic Interventions, and Modeling, 10135: 101-351L. https://doi.org/10.1117/12.2254621

[36] Cheng, R., Roth, H. R., Lay, N., Lu, L., Turkbey, B. I., Gandler, W., McCreedy, E. S., Choyke, P., Summers, R. M., \& McAuliffe, M. J. (2017). Automatic MR prostate segmentation by deep learning with holistically-nested networks, SPIE Medical Imaging, International Society for Optics and Photonics. https://doi.org/10.1117/12.2254558

[37] Gummeson, A., Arvidsson, I., Ohlsson, M., Overgaard, N. C., Krzyzanowska, A., Heyden, A., \& Aström, K. (2017) Automatic Gleason grading of H and E stained microscopic prostate images using deep convolutional neural networks. In Medical Imaging 2017: Digital Pathology,10140: p. 101400S. https://doi.org/10.1117/12.2253620

[38] Källén, H., Molin, J., Heyden, A., Lundström, C., \& Åström, K. (2016, April). Towards grading gleason score using generically trained deep convolutional neural networks. In 2016 IEEE 13th International Symposium on Biomedical Imaging (ISBI), April 2016, IEEE, pp. 1163-1167. https://doi.org/10.1109/ISBI.2016.7493473

[39] Cuocolo, R., Cipullo, M. B., Stanzione, A., Ugga, L., Romeo, V., Radice, L., \& Imbriaco, M. (2019). Machine learning applications in prostate cancer magnetic resonance imaging. European radiology experimental, 3: 1-8. https://doi.org/10.1186/s41747-019-0109-2

[40] Anwar, S. M., Majid, M., Qayyum, A., Awais, M., Alnowami, M., \& Khan, M. K. (2018). Medical image analysis using convolutional neural networks: a review. Journal of medical systems, 42: 1-13. https://doi.org/10.1007/s10916-018-1088-1

[41] Saba, T., Khan, M. A., Rehman, A., \& Marie-Sainte, S. L. (2019). Region extraction and classification of skin cancer: A heterogeneous framework of deep CNN features fusion and reduction. Journal of medical systems, 43: 1-19. https://doi.org/10.1007/s10916-019-1413-3 
[42] Premaladha, J., \& Ravichandran, K. S. (2016). Novel approaches for diagnosing melanoma skin lesions through supervised and deep learning algorithms. Journal of medical systems, 40: 1-12. https://doi.org/10.1007/s10916-016-0460-2

[43] Aima, A., \& Sharma, A. K. Predictive Approach for Melanoma Skin Cancer Detection using CNN. In Proceedings of International Conference on Sustainable Computing in Science, Technology and Management (SUSCOM), Amity University Rajasthan, Jaipur-India, February 2019. https://doi.org/10.2139/ssrn.3352407

[44] Nanditha, B. R., Geetha, A., Chandrashekar, H. S., Dinesh, M. S., \& Murali, S. (2020). Oral Malignancy Detection Using Color Features from Digital True Color Images. International Journal of Online \& Biomedical Engineering, 16(14): 95-106. https://doi.org/10.3991/ijoe. v16i14.17429

[45] Li, Y., \& Shen, L. (2018). Skin lesion analysis towards melanoma detection using deep learning network. Sensors, 18: 556. https://doi.org/10.3390/s18020556

[46] Al-Batah, M. S. (2019). Ranked Features Selection with MSBRG Algorithm and Rules Classifiers for Cervical Cancer. International Journal of Online \& Biomedical Engineering, 15(12). https://doi.org/10.3991/ijoe.v15i12.10803

[47] Pacal, I., Karaboga, D., Basturk, A., Akay, B., \& Nalbantoglu, U. (2020). A comprehensive review of deep learning in colon cancer. Computers in Biology and Medicine, 26: 104003. https://doi.org/10.1016/j.compbiomed.2020.104003

[48] Ribeiro, E., Uhl, A., \& Häfner, M., "Colonic polyp classification with convolutional neural networks", in: Proceedings of IEEE 29th International Symposium on Computer-Based Medical Systems (CBMS), IEEE, 2016, pp. 253-258. https://doi.org/10.1109/CBMS.2016.39

[49] Tanwar, S., \& Vijayalakshmi, S. (2020). Comparative analysis and proposal of deep learning based colorectal cancer polyps classification technique. Journal of Computational and Theoretical Nanoscience, 17: 2354-2362. https://doi.org/10.1166/jctn.2020.8895

[50] Zhang, R., Zheng, Y., Mak, T. W. C., Yu, R., Wong, S. H., Lau, J. Y., \& Poon, C. C. (2017). Automatic detection and classification of colorectal polyps by transferring low-level CNN features from nonmedical domain. IEEE J. Bio-med. Health Inform, 21: 41-47. https://doi. org/10.1109/JBHI.2016.2635662

[51] Yuan, Z., IzadyYazdanabadi, M., Mokkapati, D., Panvalkar, R., Shin, J. Y., Tajbakhsh, N., \& Liang, J. (2017) Automatic polyp detection in colonoscopy videos. In Medical Imaging 2017: Image Processing, 10133: p. 101332K). I https://doi.org/10.1117/12.2254671

[52] Muljo, H. H., Perbangsa, A. S., \& Yulius, B. P. (2019). Improving early cancer detection knowledge through mobile learning application. iJOE, 15(2), 61. https://doi.org/10.3991/ ijoe.v15i02.9678

\section{Authors}

Emad Shweikeh, Department of Informatics, University of Huddersfield, Huddersfield, England. E-mail: Emad.Shweikeh@hud.ac.uk.

Joan Lu, Department of Informatics, University of Huddersfield, Huddersfield, England. E-mail: j.lu@hud.ac.uk.

Murad Al-Rajab, Department of Computer Science and Information Technology, Abu Dhabi University, Abu Dhabi, United Arab Emirates. E-mail: murad.al-rajab@, adu.ac.ae.

Article submitted 2021-10-06. Resubmitted 2021-11-13. Final acceptance 2021-11-13. Final version published as submitted by the authors. 